Sains Malaysiana 50(2)(2021): 549-557

http://dx.doi.org/10.17576/jsm-2021-5002-25

\title{
On Diameter of Subgraphs of Commuting Graph in Symplectic Group for Elements of Order Three
}

(Diameter Subgraf bagi Graf Kalis Tukar Tertib dalam Kumpulan Simplektik bagi Unsur Berperingkat Tiga)

\author{
SuZILA MOHD KaSIM \& ATHIRAH NAWAWI*
}

\begin{abstract}
Suppose $G$ be a finite group and $X$ be a subset of $G$. The commuting graph, denoted by $C(G, X)$, is a simple undirected graph, where $X \subset G$ being the set of vertex and two distinct vertices $x, y \in X$ are joined by an edge if and only if $x y=$ $y x$. The aim of this paper was to describe the structure of disconnected commuting graph by considering a symplectic group and a conjugacy class of elements of order three. The main work was to discover the disc structure and the diameter of the subgraph as well as the suborbits of symplectic groups $S_{4}(2)$ ', $S_{4}(3)$ and $S_{6}(2)$. Additionally, two mathematical formulas are derived and proved, one gives the number of subgraphs based on the size of each subgraph and the size of the conjugacy class, whilst the other one gives the size of disc relying on the number and size of suborbits in each disc.
\end{abstract}

Keywords: Commuting graph; conjugacy class; disconnected graph; symplectic group

\section{ABSTRAK}

Andaikan $G$ adalah satu kumpulan terhingga dan X adalah satu subset bagi G. Grafkalis tukar tertib, ditatatandakan dengan $C(G, X)$ adalah graf mudah tidak terarah, yang menjadi set bucu dan dua bucu berbeza $x, y \in X$ disambungkan oleh satu garisbucu jika dan hanya jika $x y=y x$. Tujuan makalah ini adalah untuk memperincikan struktur graf kalis tukar tertib tidak berkait dengan mempertimbangkan kumpulan simplektik dan kelas konjugasi dengan unsur berperingkat tiga. Kerja utama adalah untuk memperoleh struktur cakera dan diameter subgraf tersebut juga suborbit bagi kumpulan simplektik $S_{4}(2)$ ', $S_{4}(3)$ dan $S_{6}(2)$. Di samping itu, dua formula matematik diterbitkan dan dibuktikan, satu daripadanya memberikan bilangan subgraf berdasarkan kepada saiz setiap subgraf dan saiz kelas konjugasi, manakala yang satu lagi memberikan saiz cakera bergantung pada bilangan dan saiz suborbit dalam setiap cakera.

Kata kunci: Graf kalis tukar tertib; graf tidak berkait; kelas konjugasi; kumpulan simplektik

\section{INTRODUCTION}

Graph theory and group theory are eminently two distinct areas of mathematical research, each with its own terminologies defined to manipulate various characteristics, problems, and complexes that seem dissociate with each other. However, numerous situations relating to algebraic structures are best dealt by using graphs, see for instance in groups (Bates et al. 2007, 2004, 2003a, 2003b; Nawawi \& Rowley 2015; Nawawi et al. 2019), and in semigroups (Abd Ghafur \& Yousof 2012). In view of one algebraic structure namely groups, we can further understand the properties of certain groups when we represent as graphs considering that the presentations are more comprehensible and the problems are more feasible especially when breaking one whole graph representing the group - in which the size of the groups are very large, into subgraphs. In fact, the methods of combining group theory and graph theory are beneficial both in theory and in practice - assisting to validate general results on groups and particular results on unique groups. Apparently, one of the real applications is in chemistry where interactions or bonds between atoms and molecules can be seen as edges between vertices. Numerical parameters of graphs known as topological indices are also used to code the chemical structure as well as to show the chemical bond order (Wang \& Ma 2016). Whilst on the other hand, energy of graphs 
calculated based on eigen values of matrices associated with the graphs is used to approximate the total electron energy of molecules (Betten et al. 2001). Indeed, connected graphs have a significant influence in several other areas, see for instance in network theory (Loh et al. 2014), dynamic systems and complexity.

In this paper, our focused group is known as symplectic group that is one of the six families of socalled classical simple groups defined in terms of a certain form on the vector space $\mathbb{V}$. The rest of the classical simple groups are the linear group, unitary group and the three families of orthogonal groups. All of them have been introduced in the forms of groups of matrices over fields. A set $\mathbb{F}$ with two binary operations on $\mathbb{F}$, the addition and multiplication satisfying certain axioms, is known as a field. Without loss of generality, let $\mathbb{V}$ be a vector space of dimension $2 c$ over the finite field $\mathbb{F}_{v^{2 c}}$ that has $v^{2 c}$ elements. For an even number $n=2 c$, the symplectic group $S_{n}(v)$ is defined as the group containing all elements of the general linear group $G L_{n}(v)$ that preserves a non-singular symplectic form and any such matrix necessarily has determinant 1 . The order of the symplectic group is given by $\left|\boldsymbol{S}_{2 c}(v)\right|=N$, where $N=v^{c^{2}}\left(v^{2 c}-1\right)\left(v^{2 c-2}-1\right)$ $\cdots\left(v^{2}-1\right)$ (Wilson 2009). In addition to this, a vast amount of information related to the symplectic group can be found in the ATLAS (Wilson et al. 2017).

The commuting graph of finite group arose from the investigation of a finite group $G$ and the set of nonidentity elements $X=G \backslash\{1\}$ (Brauer \& Fowler 1955). The most essential outcome is if a group $G$ of even order consists of more than one class of elements of order two or simply called involutions, then the distance for any two involutions $x, y \in G$ is at most three. Additionally, the study on the commuting graph was also motivated by Fischer's work in 1971 where the vertex set $X$ consists of involutions which product of any two involutions of $X$ has order at most three, known as 3-transposition group. The examples of 3-transposition groups can be viewed on the discovery of three sporadic simple groups $F_{22}, F i_{23}$ and $\mathrm{Fi}^{\prime}{ }_{24}$. Several recent researchers (Bates et al. 2007, 2004, $2003 \mathrm{a}, 2003 \mathrm{~b}$ ) have attributed to the studies of commuting graphs for certain finite groups $G$ with conjugacy class of involutions being the vertex set. The finite groups are symmetric group, Coxeter groups, special linear groups, and sporadic simple groups, respectively. Then, the venture of commuting graph was then extended to dealing with elements of larger order - conjugacy class of elements of order three to be the vertices of commuting graph (Nawawi \& Rowley 2015; Nawawi et al. 2019). All these studies specifically concentrate on the connectivity of commuting graphs - either connected or disconnected. For the case of connected commuting graphs, the diameter and the structure of each discs in commuting graph are presented in detail.

For the majority of cases of commuting graph, $G$ acts upon $X$ by conjugation and induces graph automorphisms of $C(G, X)$. Besides, $G$ is transitive permutation representation on the set of vertices $X$. The graph $C(G, X)$ is constructed by a set of vertices $X=t^{G}$ and note that $t \in G$ is chosen arbitrarily to be an initial vertex of $C(G, X)$. For $t \in G$, we define the $i^{\text {th }}$ disc of $t, \Delta_{i}(t)(i \in$ $\mathbb{N})$, which is $\Delta_{i}(t)=\{x \in X \mid d(t, x)=i\}$ where $d($, $)$ is the usual distance metric on $C(G, X)$. The diameter of $C(G$, $X)$ is denoted by Diam $C(G, X)$. In this paper, the vertices of $C(G, X)$ are the elements of order three in one of the symplectic groups $G$, either $S_{4}(2)^{\prime}, S_{4}(3)$ or $S_{6}(2)$. Without loss of generality, $G$ is assumed to be represented by its smallest degree of permutation representation and the cycle type of $t$ is $3^{r} 1^{n-3 r}$, where $r \in \mathbb{N}$ is the number of -cycles in. Thus, it is expressed by

$t=t_{1} t_{2} \cdots t_{r}=\left(a_{1,1}, a_{1,2}, a_{1,3}\right)\left(a_{2,1}, a_{2,2}, a_{2,3}\right) \cdots\left(a_{r-1,1}, a_{r-1,2}\right)$, $\left.a_{r-1,3}\right)\left(a_{r, 1}, a_{r, 2}, a_{r, 3}\right)$.

Throughout the paper, notations used for the names of conjugacy class follow the ATLAS's conventions (Wilson et al. 2017).

This paper is organized as follows. The next section begins with some details of how the calculations were performed as well as introducing relevant notations. The following section presents the tabulated data on the diameters and the orbit structures of subgraph $D(G, X)$ of disconnected $C(G, X)$ in Tables 1 and 2 and provides some general results in accordance to the properties of certain elements in the connected component of disconnected $C(G, X)$. Finally, a conclusion about the whole research is drawn in the last section.

\section{MATERIALS AND Methods}

This paper considers all graphs to be simple, finite, and undirected. A graph $\Gamma$ is built with the set of vertices $V$ and the set of edges $E$. Two vertices $u, v \in V$ are adjacent if there is an edge joining them. A graph $\Gamma$ is connected if for any two vertices $u, v \in V$ are linked by a path in $\Gamma$, otherwise the graph is disconnected. The distance between vertices $u, v \in \Gamma$ or the length of a shortest path is written as $d(u, v)$. Then, $\operatorname{Diam}(\Gamma)=\max \{\mathrm{d}(\mathrm{u}, \mathrm{v}) \mid \mathrm{u}, \mathrm{v} \in V\}$. The degree of $u \in V, \operatorname{deg}(u)$ is the number of edges incident with $u$. Let $\Gamma_{1}$ and $\Gamma_{2}$ be two graphs with $\mathrm{V}_{1} \subseteq \mathrm{V}_{2}$ and $\mathrm{E}_{1}$ $\subseteq \mathrm{E}_{2}$, respectively, then $\Gamma_{1}$ is a subgraph of $\Gamma_{2}$ (Malik et al. 2014).

Recall that, for $G$ a symplectic group either $S_{4}(2)$ ', $S_{4}(3)$ or $S_{6}(2)$, and $X$ a $G$-conjugacy class of elements of order $p=3$. The calculations will be carried out using the smallest permutation representation for $G$ supplied by the 
ATLAS together with the aid of algebra package MAGMA (Bosma et al. 1997). For the commuting graph $C(G, X)$, an element $t \in G$ is fixed, and then the size of $\Delta_{i}(t)$ on $X$ and Diam $C(G, X)$ are determined. Clearly, when $t$ is adjacent to some elements $X \in X$, it will be linked by edges since $t x=x t$. All neighbours of $t$ are now located in the first disc $\Delta_{1}(t)$ which shows $d(t, x)=1$ for $x \in \Delta_{1}(t)$. Despite the fact that some elements $y \in \Delta_{1}(t)$ might commute with some other elements in the same disc, they will also be connected to the rest of elements $x \in X \mid \Delta_{1}(t)$ such that $y x$ $=x y$. Therefore, all the elements are put in the second disc $\Delta_{2}(t)$. This process repeated and will be stopped until the last disc of $t$. However, if $|t|+\sum_{i}\left|\Delta_{i}(t)\right| \neq|X|$, then the graph $C(G, X)$ is actually disconnected. Thus, the elements with the size $|t|+\sum_{i}\left|\Delta_{i}(t)\right|$ are putting in a component or subgraph, called $D(G, X)$.

Under the action of the centralizer of $t$ in $G$, denoted by $C_{G}(t)$, the discs $\Delta_{i}(t)$ are broken into smaller understandable parts called suborbits or we call them as $C_{G}(t)$-orbits. An arbitrary element $x \in X$ is selected to represent each $C_{G}(t)$-orbit to be a $C_{G}(t)$-orbit representative. The interest in identifying the size of each $C_{G}(t)$-orbit of is to compute the size of centralizer $C_{C_{G}(t)}\langle t, x\rangle$.

Now, for each $C_{G}(t)$-orbit, the focus is on the subgroup $H=\langle t, x\rangle$ that is generated by $t, x \in X$. Let $m$ to be the order of the product $t x \in X$ and $m \|\langle t, x\rangle \mid$. Up to isomorphisms, the group that is isomorphic to $H$ is identified. The cases of the abelian groups are done by comparing the orders of elements in $H$. If $H$ are found to be non-abelian and non-simple groups, a group extension is solved by breaking down a group as a certain combination of simple groups. The group extension can be classified by letting $N$ and $F$ be two groups, then $H^{\prime}$ is an extension of $F$ by $N$ if there is an exact sequence $1 \rightarrow N \rightarrow H^{\prime} \rightarrow F \rightarrow 1$. Clearly, $H^{\prime}$ is a group, $N$ is a normal subgroup of $H^{\prime}$ and the group $F$ is isomorphic to the quotient group $H^{\prime} / N$. Then, $H^{\prime}$ is decided to be either $H^{\prime}=N \times F$ (the direct product),
$H^{\prime}=N: F($ the split extension of $N$ and $N)$, or $H^{\prime}=N \cdot F$ (the non-split extension of $N$ and $F$ ).

\section{RESULTS AND DISCUSSION}

This section is begun by providing the supplementary notations and preliminaries. Let $\Omega=\{1,2, \ldots, n\}$ and $\operatorname{Sym}(\Omega)=\operatorname{Sym}(n)$. For $t \in G$ and $x$ is any other permutation in $X, \operatorname{supp}(x)$ is denoted as the set of points of $\Omega$ not fixed by $X$. Suppose two sets $X$ and $Y$ in $G$ and $X \subseteq Y$ which simply means either $X$ is contained inside $Y$ or $X=Y$. Besides, $\Lambda$ and $\Delta$ are given as $\Lambda=\operatorname{supp}(t) \cap \operatorname{supp}(x)$ and $\Delta=\operatorname{supp}(t) \cup \operatorname{supp}(x)$. Now, let $i, j, k \in \mathbb{N}$ and assume $x$ to be one or combinations of these products - the product of 3-cycles $\alpha_{i}$ which are left uncycled or being inverted, the product of 3 -cycles $\rho$. which are obtained by cycling disjoint sets of three 3 -cycles of $t$ and the product of 3 -cycles $\sigma_{\mathrm{k}}$ with $\operatorname{supp}\left(\sigma_{\mathrm{k}}\right)=\Omega \backslash \operatorname{supp}(t)$, in whichi, $j, k \in \mathbb{N}$ are actually the lengths of the product of pairwise disjoint 3 -cycles of $t$.

Let $G$ be a symplectic group and $X$ is a $G$-conjugacy class for $p=3$. By applying the methods explained in previous section on MAGMA (Bosma et al. 1997), observe that $C(G, X)$ is disconnected when:

$G=S_{4}(2)^{\prime}$ and $X=3 A, 3 B, 3 \mathrm{AB} ; G=S_{4}(3)$ and $X=3 C, 3 D$; $G=S_{6}(2)$ and $X=3 B, 3 C$.

Moreover, let $D(G, X)$ be the connected component of $C(G, X)$, then $D(G, X)$ is also subgraph of $C(G, X)$. Then, the diameters and the orbit structures of subgraph $D(G$, $X)$ for $p=3$ are presented in Tables 1 and 2, respectively.

These analyses also include other important measures such as $|D(G, X)|$ - the sizes of subgraphs $D(G$, $X)$ of $C(G, X),\left|\Delta_{i}(t)\right|$ - the sizes of $\Delta_{i}(t), N_{D}$ - the numbers of subgraphs $D(G, X)$ in $C(G, X), t x \in X$ - the conjugacy class of the product $t \in G$ and $x \in X,\left|\mathcal{O}_{i}(t)\right|$ - the sizes of $C_{G}(t)$-orbits in each $\Delta_{i}(t)$ and $\langle t, x\rangle$ - the subgroups generated by $t \in G$ and $x \in X$, as well as their orders.

TABLE 1 . The diameters of each $D(G, X)$

\begin{tabular}{cccccccc}
\hline$G$ & $X$ & $\left|C_{G}(t)\right|$ & $|X|$ & $|D(G, X)|$ & $\left|\Delta_{1}(t)\right|$ & $\left|\Delta_{2}(t)\right|$ & $N_{D}$ \\
\hline$S_{4}(2)^{\prime}$ & $3 A$ & 9 & 40 & 4 & 3 & - & 10 \\
& $3 B$ & 9 & 40 & 4 & 3 & - & 10 \\
& $3 A B$ & 18 & 80 & 8 & 7 & - & 10 \\
$S_{4}(3)$ & $3 C$ & 108 & 240 & 6 & 5 & - & 40 \\
& $3 D$ & 54 & 480 & 12 & 11 & - & 40 \\
$S_{6}(2)$ & $3 B$ & 648 & 2240 & 80 & 25 & 54 & 28 \\
& $3 C$ & 108 & 13440 & 12 & 11 & - & 1120 \\
\hline
\end{tabular}


TABLE 2. The orbit structures of each $D(G, X)$

\begin{tabular}{|c|c|c|c|c|c|c|c|}
\hline$G$ & $X$ & $\Delta_{i}(t)$ & $t x \in X$ & $N_{O i(t)}$ & $\left|O_{i}(t)\right|$ & $|\langle t, x\rangle|$ & $\langle t, x\rangle$ \\
\hline \multirow[t]{6}{*}{$S_{4}(2)^{\prime}$} & $3 A$ & $\Delta_{1}(t)$ & $1 A$ & 1 & 1 & 3 & $A_{3}$ \\
\hline & & & $3 B$ & 2 & 1 & 9 & $\mathbb{Z}_{3} \times \mathbb{Z}_{3}$ \\
\hline & $3 B$ & $\Delta_{1}(t)$ & $1 A$ & 1 & 1 & 3 & $A_{3}$ \\
\hline & & & $3 A$ & 2 & 1 & 9 & $\mathbb{Z}_{3} \times \mathbb{Z}_{3}$ \\
\hline & $3 A B$ & $\Delta_{1}(t)$ & $1 A$ & 1 & 1 & 3 & $A_{3}$ \\
\hline & & & $3 A B$ & 3 & 2 & 9 & $\mathbb{Z}_{3} \times \mathbb{Z}_{3}$ \\
\hline \multirow[t]{7}{*}{$S_{4}(3)$} & $3 C$ & $\Delta_{1}(t)$ & $1 A$ & 1 & 1 & 3 & $A_{3}$ \\
\hline & & & $3 D$ & 1 & 4 & 9 & $\mathbb{Z}_{3} \times \mathbb{Z}_{3}$ \\
\hline & $3 D$ & $\Delta_{1}(t)$ & $1 A$ & 1 & 1 & 3 & $A_{3}$ \\
\hline & & & $3 A$ & 1 & 2 & 9 & $\mathbb{Z}_{3} \times \mathbb{Z}_{3}$ \\
\hline & & & $3 B$ & 1 & 2 & 9 & $\mathbb{Z}_{3} \times \mathbb{Z}_{3}$ \\
\hline & & & $3 C$ & 1 & 2 & 9 & $\mathbb{Z}_{3} \times \mathbb{Z}_{3}$ \\
\hline & & & $3 D$ & 2 & 2 & 9 & $\mathbb{Z}_{3} \times \mathbb{Z}_{3}$ \\
\hline \multirow[t]{9}{*}{$S_{6}(2)$} & $3 B$ & $\Delta_{1}(t)$ & $1 A$ & 1 & 1 & 3 & $A_{3}$ \\
\hline & & & $3 A$ & 1 & 12 & 9 & $\mathbb{Z}_{3} \times \mathbb{Z}_{3}$ \\
\hline & & & $3 C$ & 1 & 12 & 9 & $\mathbb{Z}_{3} \times \mathbb{Z}_{3}$ \\
\hline & & $\Delta_{1}(t)$ & $4 A$ & 1 & 27 & 24 & $Q_{8}: \mathbb{Z}_{3}$ \\
\hline & & & $6 \mathrm{C}$ & 1 & 27 & 24 & $Q_{8}: \mathbb{Z}_{3}$ \\
\hline & $3 C$ & $\Delta_{1}(t)$ & $1 A$ & 1 & 1 & 3 & $A_{3}$ \\
\hline & & & $3 A$ & 1 & 2 & 9 & $\mathbb{Z}_{3} \times \mathbb{Z}_{3}$ \\
\hline & & & $3 B$ & 1 & 4 & 9 & $\mathbb{Z}_{3} \times \mathbb{Z}_{3}$ \\
\hline & & & $3 C$ & 1 & 4 & 9 & $\mathbb{Z}_{3} \times \mathbb{Z}_{3}$ \\
\hline
\end{tabular}

Lemma 1 Let $t \in G$ be an element of order three of cycle type $3^{r} 1^{n-3 r}$ and $x \in X=t^{G}$ (or simply an element in the same conjugacy class as $t$ ), then $t$ commutes with some of the following observations:

$x=\alpha_{1} \alpha_{2} \ldots \alpha_{i}$, the product of $r=i$ pairwise disjoint 3-cycles of $t ; x=\alpha_{1} \alpha_{2} \ldots \alpha_{i} \rho_{1} \rho_{2} \ldots \rho_{j}$, the product of $r=i+j$ pairwise disjoint 3-cycles of , the product of pairwise disjoint 3 -cycles of $t$; and $x=\rho_{1} \rho_{2} \ldots \rho_{j} \sigma_{1} \sigma_{2} \ldots \sigma_{k}$, the product of $r=j+k$ pairwise disjoint 3-cycles of $t$.
Proof Suppose $x=\alpha_{1} \alpha_{2} \ldots \alpha_{i}$, the product of $r=i$ pairwise disjoint 3-cycles of $t$. By the definition, the product of $\alpha_{i}$ are the 3-cycles of which are left uncycled or being inverted. Clearly, $\operatorname{supp}(t)=\operatorname{supp}(x)$ and $\Lambda=\Delta$. Furthermore, $x=t^{-1}$ is one of the elements in $X$ which has the required property where all of 3-cycles of are being inverted and consequently $t x=x t$.

Let $x=\rho_{1} \rho_{2} \ldots \rho_{j}$ be the product of $r=j$ pairwise disjoint 3 -cycles of $t$. It is defined as the product of 3-cycles $\rho_{j}$ 
which are obtained by cycling disjoint sets of three 3-cycles of $t$. Hence there is a connection between $t, x \in X$ due to $\operatorname{supp}(t)=\operatorname{supp}(x)$ and $\Lambda=\Delta$. Let say $t=t_{1} t_{2} \ldots t_{r}$, then any three disjoint 3-cycles of $t$ are chosen such that $t_{1} t_{2} t_{3}$ $=\left(a_{1,1}, a_{1,2}, a_{1,3}\right)\left(a_{2,1}, a_{2,2}, a_{2,3}\right)\left(a_{3,1}, a_{3,2}, a_{3,3}\right)$ where $\left\{a_{1,1}\right.$, $\left.a_{1,2}, a_{1,3}\right\} \in t_{1},\left\{a_{2,1}, a_{2,2}, a_{2,3}\right\} \in t_{2}$ and $\left\{a_{3,1}, a_{3,2}, a_{3,3}\right\} \in t_{3}$. For any three disjoint 3 -cycles of $x=\rho_{1} \rho_{2} \ldots \rho_{j}$, consider $\rho_{1} \rho_{2} \rho_{3}=\left(b_{1,1}, b_{1,2}, b_{1,3}\right)\left(b_{2,1}, b_{2,2}, b_{2,3}\right)\left(b_{3,1}, b_{3,2}, b_{3,3}\right)$, with $\left.\left\{b_{1,1}, b_{1,2}, b_{1,3}\right\} \in \rho_{1}, b_{2,1}, b_{2,2}, b_{2,3}\right\} \in \rho_{2}$ and $\left.b_{3,1}, b_{3,2}, b_{3,3}\right\} \in$ $\rho_{3}$. In making sure $\operatorname{supp}\left(t_{1} t_{2} t_{3}\right)=\operatorname{supp}\left(\rho_{1} \rho_{2} \rho_{3}\right)$, note that $s_{*}$ $=\operatorname{supp}\left(t_{1} t_{2} t_{3}\right) \cap \operatorname{supp}\left(\rho_{1} \rho_{2} \rho_{3}\right)$ with $\left|S_{*}\right|=9$ and $\operatorname{supp}\left(t_{1} t_{2} t_{3}\right)$ $\mathrm{U} \operatorname{supp}\left(\rho_{1} \rho_{2} \rho_{3}\right)$.

For $x=\alpha_{1} \alpha_{2} \ldots \alpha_{i} \rho_{1} \rho_{2} \ldots \rho_{i}$, the product of $r=i+j$ pairwise disjoint 3-cycles of $t$. Following the same argument as in Part 1 and Part 2, $\operatorname{supp}(t)=\operatorname{supp}(x)$ and $\Lambda=\Delta$, then $t, x \in$ $X$ commute with each other.

Suppose $x=\rho_{1} \rho_{2} \ldots \rho_{j} \sigma_{1} \sigma_{2} \ldots \sigma_{k}$ be the product of $r=j$ $+k$ pairwise disjoint 3-cycles of $t$. With the existence of $k$ pairwise disjoint 3-cycles of $\sigma_{1} \sigma_{2} \ldots \sigma_{k}$, then $\operatorname{supp}(t) \neq$ $\operatorname{supp}(x)$ and $\Lambda \subseteq \Delta$. Furthermore, $t$ satisfies $\operatorname{supp}(t) \subseteq \Omega$ and it is proved that $t, x \in X$ commute.

In the next result, any three disjoint 3-cycles of are focused such that $t_{1} t_{2} t_{3}=\left(a_{1,1}, a_{1,2}, a_{1,3}\right)\left(a_{2,1}, a_{2,2}, a_{2,3}\right)\left(a_{3,1}\right.$, $\left.a_{3,2}, a_{3,3}\right)$ where $\left.a_{1,1}, a_{1,2}, a_{1,3}\right\} \in t_{1},\left\{a_{2,1}, a_{2,2}, a_{2,3}\right\} \in t_{2}$ and $\left\{a_{3,1}, a_{3,2}, a_{3,3}\right\} \in t_{3}$.

Theorem 1 Suppose that $D(G, X)$ denotes one of the subgraph in the disconnected $C(G, X)$. Then, $D(G, X)$ is isomorphic to each other. Thus, the number of subgraphs equals the size of each subgraph divides the size of the conjugacy class $X, N_{D}=\frac{|X|}{|D(G, X)|}$

Proof Note that $t$ is of cycle type $3^{r} 1^{n-3 r}$ where $r \in \mathbb{N}, X=$

$t^{G}$ and $G \leq \operatorname{Sym}(\Omega)(\operatorname{or} \operatorname{Sym}(n))$. Let $q=\Omega \backslash \operatorname{supp}(t)$. If $n=$ $3 r+q$ then, $t$ fixes only $q$ points. Clearly, any conjugate of $t(x \in X)$ that commute with $t$ will also fix $q$ points. So does every conjugate of $t$ contained in the subgraph of $C(G, X)$. Hence $C(G, X)$ is disconnected. In other words, all subgraphs $D(G, X)$ are isomorphic of size $h \in \mathbb{N}$. In order to compute the number of subgraph in the disconnected $C(G$, $X)$, every subgraph contains elements of $X$ which giving a path $(t, x)$ in $D(G, X)$. As $G$ is transitive on the vertices of $C(G, X)$, then this simply contributes the formula

$$
N_{D}=\frac{|X|}{|D(G, X)|} .
$$

Lemma 2 Assume that $t \in G$ be an element of order three and $i \in \mathbb{N}$ then, $x \in \Delta_{i}(t)$ if the following observations hold:

$$
\begin{aligned}
& \text { 1. } x \in \Delta_{1}(t) \text { and }|\langle t, x\rangle|=3 \text {, then } H=\langle t, x\rangle \cong A_{3} . \\
& \text { 2. } x \in \Delta_{1}(t) \text { and }|\langle t, x\rangle|=9 \text {, then }\langle t, x\rangle \cong \mathbb{Z}_{3} \times \mathbb{Z}_{3} . \\
& \text { 3. } x \in \Delta_{2}(t) \text { and }|\langle t, x\rangle|=24 \text {, then }\langle t, x\rangle \cong Q_{8}: \mathbb{Z}_{3} .
\end{aligned}
$$

Proof The fact that the subgroup $H=\langle t, x\rangle$ is of order three which is a prime number, then there is a unique, up to isomorphism, a group of prime order named as the cyclic group. Clearly, $H \cong \mathbb{Z}_{3} \cong A_{3}$ which has the presentation $\left\langle t, x \mid t^{3}=x^{3}=t x=1\right\rangle$ and contains elements of orders 1 and 3. Additionally, the subgroup $H$ is an elementary abelian and simple group.

For this case, the subgroup $H=\langle t, x\rangle$ is of order 9. Clearly, there are only two possibilities of groups having order 9 , either it is a cyclic group $\mathbb{Z}_{9}$, or a direct product of two cyclic groups, $\mathbb{Z}_{3} \times \mathbb{Z}_{3}$. Knowing that $t$ and $x$ are both elements of conjugacy class of order three and the order of $t x$ is also three in which consequently $H$ can have the presentation $\left\langle t, x \mid t^{3}=x^{3}=(t x)^{3}=1\right\rangle$, the latter candidate of group of order 9 . The subgroup $\mathbb{Z}_{3} \times \mathbb{Z}_{3}$ is an elementary abelian, non-cyclic and non-simple group.

The subgroup $H=\langle t, x\rangle$ is of order $24=2^{3} \cdot 3$. Undeniably, there are 15 candidates of small groups of order 24 and both $t$ and $x$ are elements of conjugacy class of order three and the product $t x$ is either element of class $4 A$ or $6 C$. Note that $H$ is non-abelian, non-simple and solvable group. In order to guess which group is isomorphic to $H$, assume that $N$ be a normal subgroup of $H$ which is isomorphic to a quartenion group of order $8, Q_{8}$. Then, the corresponding factor group $F=H / N$ is determined as a cyclic group of order 3 . Moreover, we can tackle this case by using another normal subgroup $N^{\prime}$ of $S \in \operatorname{Syl}_{2}(H)$ with factor group $R=S / N^{\prime}$ such that $N^{\prime} R=S$ and $N^{\prime} \cap$ $R$. There exists an identity of group in $S / N^{\prime}$ to show that $S$ splits over $N^{\prime}$, so does $H$. Therefore, $H \cong Q_{8}: \mathbb{Z}_{3}$, a split extension of a cyclic group of order 3 by $Q_{8}$.

Theorem 2 Let $N_{\text {Oi(t) }}$ be the number of $C_{C_{G}(t)}$-orbit in each $\Delta_{i}(t)$. Then we have

$$
\left|\Delta_{i}(t)\right|=N_{O_{i}(t)}\left(\frac{\left|C_{G}(t)\right|}{\left|C_{\left.G_{G^{(t)}}(t, x)\right)}\right|}\right)
$$

Proof In order to obtain which disc of $t$ contain the vertices of $X$, we may utilize the size of $\Delta_{i}(t)$ to unite certain of

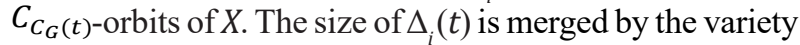
size of $C_{C_{G}(t)}$-orbits where $\left|\mathcal{O}_{i}(t)\right|=\left|C_{G}(t)\right| /\left|C_{C_{G}(t)}(\langle t, x\rangle)\right|$. $\left|C_{G}(t)\right|$ is divided by $\left|C_{C_{G}(t)}(\langle t, x\rangle)\right|$, instead of considering $C_{C_{G}(t)}$ (x) (Nawawi 2013). Simultaneously, $\left|\mathrm{O}_{i}(t)\right|$ are positioned together with $N_{\mathrm{O} i(t)}$ of the same size of the $C_{G}(t)$-orbit. So, we formulate $\Delta_{i}(t)=N_{\text {Oi(t) }}\left(\left|C_{G}(t)\right| / \mid C_{C_{G}(t)}\right.$ $(\langle t, x\rangle) \mid)$.

One of the features in studying of commuting graph is that it can be represented pictorially where we can draw a diagram so-called the collapsed adjacency diagram. Let $\Delta_{\ell}^{k}(t)$ and $\Delta_{\ell}^{k \prime}(t)$ are two $C_{G}(t)$-orbits of $X$. A collapsed adjacency diagram defines the interactions between these 
orbits. Figure 1 considers a circle to denote an orbit with a name within the circle. A line together with an arrow on one end of the line is drawn from an orbit to other orbit, thus it displays some edges exist between vertices in these orbits. A vertex in the orbit $\Delta_{\ell}^{k}(t)$ may be connected to $\epsilon$ vertices in $\Delta_{\ell}^{k}(t)$, and to $\alpha$ vertices in $\Delta_{\ell \prime}^{k^{\prime}}(t)$. Then, a vertex in the orbit $\Delta_{\ell}^{k \prime}(t)$ is connected to $\epsilon^{\prime}$ vertices in $\Delta_{\ell \prime}^{k \prime}(t)$, and to $\alpha^{\prime}$ vertices in $\Delta_{\ell}^{k}(t)$.

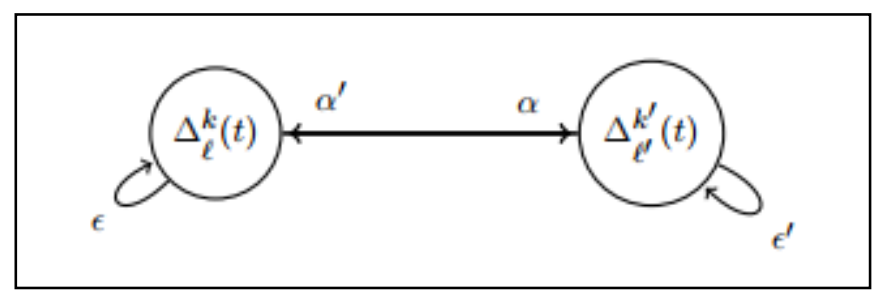

FIGURE 1. Interactions of orbits in a collapsed adjacency diagram

If every $C_{G}(t)$-orbit contains only one vertex, then that orbit is illustrated by a small black circle without any labeling as in Figure 2.

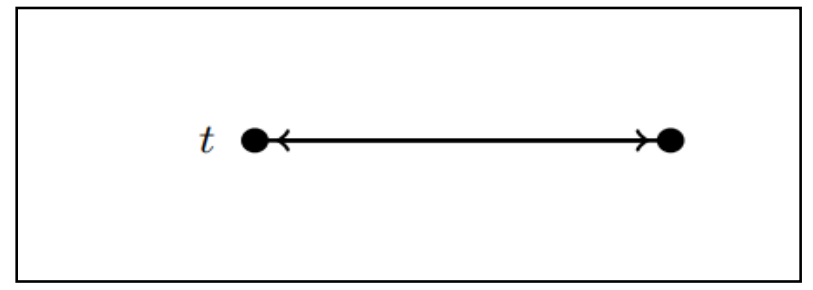

FIGURE 2. Interactions of trivial orbits in a collapsed adjacency diagram

Example 1 By referring to ATLAS (Wilson et al. 2017), symplectic group $S_{4}(2)^{\prime}$ is isomorphic to $L_{2}(9)$, the projective special linear group of degree two over field $\mathbb{F}_{9}$, and also to $A_{6}$, the alternating group of degree 6 . From now, for the purpose of computation, let $S_{4}(2)^{\prime} \cong A_{6}$ which are generated by $a=(1,2)(3,4)$ and $\mathrm{b}=(1,2,3,5)(4,6)$. Consider the first conjugacy class of element of order three in $S_{4}(2)^{\prime}$ which is denoted by $3 A$. Assume $t$ now as $3 A$ class representative which is given as the product $a b a b a b^{-1} a b^{2}$ which is actually $t=(1,6,3)$ (Wilson et al. 2017), an element of cycle type $3^{1} 1^{3}$. Now, observing the conjugacy class $3 A$ :

$$
\begin{aligned}
X= & 3 A=t^{G}=(1,6,3)^{G} \\
X=\{(1,6,3),(2,6,4),(2,4,5),(3,4,6),(1,3,5),(1,3,6),(3,6,4),(4,5,6),(1,2,5), & \\
& (2,4,6),(2,5,4),(4,6,5),(3,5,6),(1,4,6),(1,5,2),(1,2,3),(1,5,3),(3,6,5), \\
& (1,6,4),(1,4,5),(2,3,6),(1,3,2),(1,4,2),(2,3,5),(1,5,4),(2,6,3),(1,2,6), \\
& (3,5,4),(1,2,4),(2,5,3),(1,6,2),(3,4,5),(2,3,4),(1,6,5),(2,4,3),(1,5,6), \\
& (1,4,3),(2,6,5),(1,3,4),(2,5,6)\} .
\end{aligned}
$$


Clearly, $t=(1,6,3)$ commutes with only three elements in $X$, that is $t^{-1}=(1,3,6)$, which satisfying Lemma 1 (1), and also with $(2,4,5)$ and $(2,5,4)$, which satisfying Lemma 1 (4). Hence, it is now confirmed that the commuting graph $C\left(S_{4}(2)^{\prime}, 3 A\right)$ is disconnected and we may set the vertex set of first subgraph (or component) of $C\left(S_{4}(2)^{\prime}, 3 A\right)$ to be:

$$
V\left(D_{1}\left(S_{4}(2)^{\prime}, 3 A\right)\right)=\{(1,6,3),(1,3,6),(2,4,5),(2,5,4)\} .
$$

The subgraph $D_{1}\left(S_{4}(2)^{\prime}, 3 A\right)$ can be viewed as a complete graph $K_{4}$ of diameter 1 as shown in Figure 3:

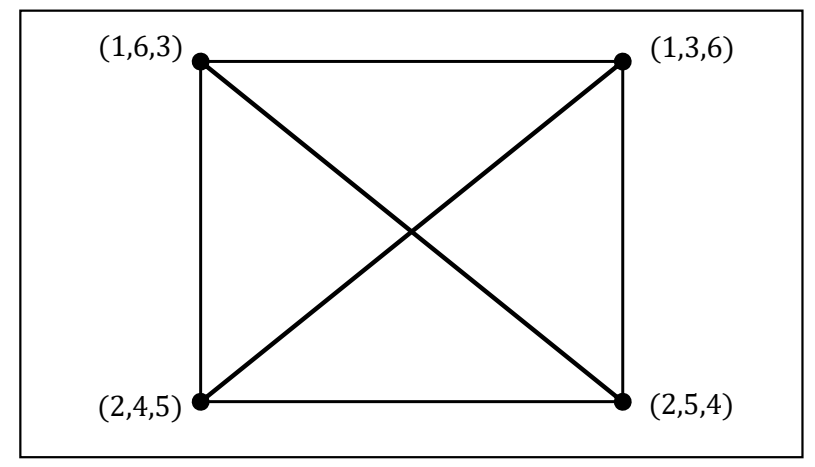

FIGURE 3. The subgraph $D_{1}\left(S_{4}(2)^{\prime}, 3 A\right)$

In order to construct another subgraph (or component) of $C\left(S_{4}(2)^{\prime}, 3 A\right)$, we may choose, without loss of generality, an element $X$ in which is not in $V\left(D_{1}\left(S_{4}(2)^{\prime}, 3 A\right)\right.$, say $x=(1,2,5)$. Obviously, we can have the vertex set of second subgraph (or component) of
$C\left(S_{4}(2)^{\prime}, 3 A\right)$ to be:

$$
V\left(D_{2}\left(S_{4}(2)^{\prime}, 3 A\right)\right)=\{(1,2,5),(1,5,2),(3,4,6),(3,6,4)\} .
$$

The subgraph $D_{2}\left(S_{4}(2)^{\prime}, 3 A\right)$ also can be viewed as a complete graph $K_{4}$ of diameter 1 as in Figure 4:

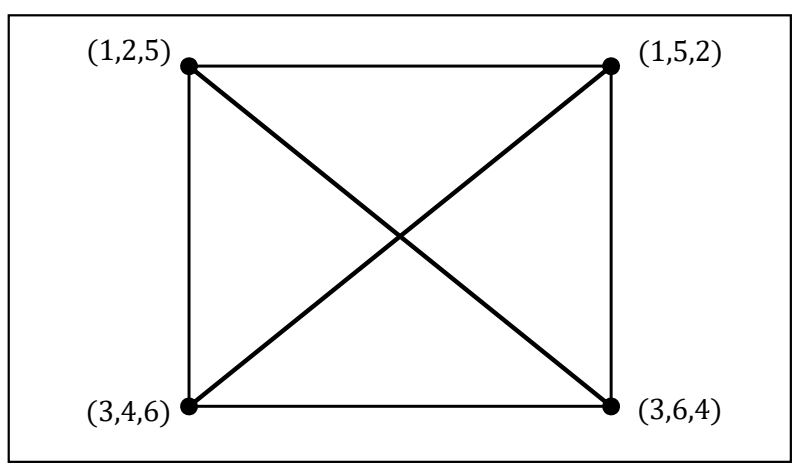

FIGURE 4. The subgraph $D_{2}\left(S_{4}(2)^{\prime}, 3 A\right)$

Thus, the subgraph $D_{1}\left(S_{4}(2)^{\prime}, 3 A\right)$ is isomorphic to the subgraph $D_{2}\left(S_{4}(2)^{\prime}, 3 A\right)$ and in fact, if we proceed for the rest of elements in $X=3 A$, we will get the isomorphism between all subgraphs of $C\left(S_{4}(2)^{\prime}, 3 A\right)$. Therefore, the number of subgraphs $N_{D}$ can be obtained by dividing the size of $X$ and the size of each subgraph as shown below:

$$
N_{D}=\frac{|X|}{\left|V\left(D\left(S_{4}(2)^{\prime}, 3 A\right)\right)\right|}=\frac{40}{4}=10
$$


conforming the information given in Table 1 and consequently satisfying Theorem 1 .

Now, dealing with elements in $V\left(D_{1}\left(S_{4}(2)^{\prime}, 3 A\right)\right.$, say $t$ $=(1,6,3)$ and $x_{1}=(1,3,6)$, the product $t x_{1}=(1) \in 1 A$ whilst if $x_{2}=(2,4,5)$ and $x_{3}=(2,5,4)$, their products $t x_{2}=(1,6,3)$ $(2,4,5)$ and $t x_{3}=(1,6,3)(2,5,4)$ are both elements in $3 B$ (a conjugacy class of element of order three and the cycle type of its elements is $3^{2}$ ).

Additionally, the subgroup generated by $t=(1,6,3)$ and $x_{1}=(1,3,6)$, denoted by $\left\langle t, x_{1}>\right.$, can be described below:

$$
<t, x_{1}>=<(1,6,3),(1,3,6)>=\{(1),(1,6,3),(1,3,6)\}
$$

which is isomorphic to the alternating group $A_{3}$, thus satisfying Lemma 2(1).

Furthermore, the subgroup generated by $t=(1,6,3)$ and $x_{2}=(2,4,5)$, denoted by $\left\langle t, x_{2}\right\rangle$, can be described below:

$$
\begin{aligned}
<\mathrm{t}, \mathrm{x}_{2}>= & <(1,6,3),(2,4,5)> \\
= & \{(1),(1,6,3),(1,3,6),(2,4,5),(2,5,4),(1,6,3) \\
& (2,4,5),(1,6,3)(2,5,4),(1,3,6)(2,4,5), \\
& (1,3,6)(2,5,4)\}
\end{aligned}
$$

which is isomorphic to the direct product of an elementary abelian group $Z_{3}$ with itself or simply written as $\mathbb{Z}_{3} \times \mathbb{Z}_{3}$, thus satisfying Lemma $2(2)$. Therefore, $D\left(S_{4}(2)\right.$ ', $\left.3 A\right)$ breaks into $3 C_{G}(t)$-orbits in $\Delta_{1}(t)$ which supports Theorem 2 such that:

$$
\left|\Delta_{1}(t)\right|=\frac{\left|C_{G}(t)\right|}{9}+\frac{2\left|C_{G}(t)\right|}{9} .
$$

Each $C_{G}(t)$-orbit in $D\left(S_{4}(2)^{\prime}, 3 A\right)$ is of size 1 and the collapsed adjacency diagram of $D\left(S_{4}(2)^{\prime}, 3 A\right)$ is designed as in Figure 4. On the other hand, the commuting graph $C\left(S_{4}(2)^{\prime}, 3 B\right)$ also demonstrates the same properties as the commuting graph $C\left(S_{4}(2)^{\prime}, 3 A\right)$.

\section{CONCLUSION}

This research contributes to the study of commuting graph $C(G, X)$ for symplectic groups $S_{4}(2)^{\prime}, S_{4}(3)$ and $S_{6}(2)$. The graphs $C(G, X)$ considered here are all disconnected hence motivated us to determine the structure of the components or subgraphs of $C(G, X)$, denoted by $D(G$, $X)$. Our main concern is its diameter as well as the size of each discs in the subgraphs. Additionally, study of the commuting graphs leads naturally to consideration of the suborbit structure of the class $X$. Once all the suborbits have been located, we determine the subgroup $\langle t, x\rangle$ for every representative $x \in X$ in all discs $\Delta_{i}(t)$. Two mathematical formulas are also derived and proved, one gives the number of subgraphs based on the size of each subgraph and the size of the conjugacy class, whilst the other one gives the size of disc relying on the number and size of suborbits in each disc.

\section{ACKNOWLEDGEMENTS}

This research was funded by the Ministry of Higher Education (MOHE), Malaysia under the Fundamental Research Grant Scheme (FRGS/1/2019/STG06/UPM/02/9). We are also indebted to the referees for their suggestions and useful remarks.

\section{REFERENCES}

Abd Ghafur Bin Ahmad \& Yousof Gheisari. 2012. Components in graphs of diagram groups over the union of two semigroup presentations of integers. Sains Malaysiana 41(1): 129-131

Bates, C., Bundy, D., Hart, S. \& Rowley, P. 2007. Commuting involution graphs for sporadic simple groups. J. Algebra 316(2): 849-868.

Bates, C., Bundy, D., Perkins, S. \& Rowley, P. 2004. Commuting involution graphs in special linear groups. Comm. Algebra 32(11): 4179-4196.

Bates, C., Bundy, D., Perkins, S. \& Rowley, P. 2003a. Commuting involution graphs for symmetric groups. J. Algebra 266(1): 133-153.

Bates, C., Bundy, D., Perkins, S. \& Rowley, P. 2003b. Commuting involution graphs in finite Coxeter groups. J. Group Theory 6(4): 461-476.

Betten, A., Kohnert, A., Laue, R. \& Wassermann, A. 2001. Algebraic combinatorics and applications. In The Energy of a Graph: Old and New Results, edited by Gutman, I. Berlin, Heidelberg: Springer. pp. 196-211.

Bosma, W., Cannon, J.J. \& Playoust, C. 1997. The magma algebra system I: The user language. J. Symbolic Comput. 24(3-4): 235-265.

Brauer, R. \& Fowler, K.A. 1955. On groups of even order. Ann. of Math. 62(3): 565-583.

Fischer, B. 1971. Finite groups generated by 3 -transpositions. I Invent. Math. 13(3): 232-246.

Loh, S.L., Salleh, S. \& Sarmin, N.H. 2014. Linear-time heuristic partitioning technique for mapping of connected graphs into single-row networks. Sains Malaysiana 43(8): 1263-1269.

Malik, D.S., Sen, M.K. \& Ghosh, S. 2014. Introduction to Graph Theory. Singapore: Cengage Learning Asia Pte Ltd.

Nawawi, A. 2013. Commuting graphs for elements of order three in finite groups. University of Manchester. Ph.D. Thesis (Unpublished).

Nawawi, A. \& Rowley, P. 2015. On commuting graphs for elements of order 3 in symmetric groups. Elec. J. Comb. 22(1): P1.21.

Nawawi, A., Husain, S.K.S. \& Ariffin, M.R.K. 2019. Commuting graphs, $\mathrm{C}(\mathrm{G}, \mathrm{X})$ in symmetric groups $\operatorname{Sym}(n)$ and its connectivity. Symmetry 11(9): 1178.

Wang, Y-F. \& Ma, N. 2016. Orderings a class of unicyclic graphs with respect to hosoya and merrifield-simmons index. Sains Malaysiana 45(1): 55-58. 
Wilson, R.A. 2009. Graduate Texts in Mathematics: The Finite Simple Groups. London, England: Springer-Verlag. pp. 41-110.

Wilson, R.A., Walsh, P., Tripp, J., Suleiman, I., Parker, R., Norton, S., Nickerson, S., Linton, S., Bray, J. \& Abbott, R. 2017. http://brauer.maths.qmul.ac.uk/Atlas/v3/. World-wide-web atlas of finite group representations.

Suzila Mohd Kasim \& Athirah Nawawi* Institute for Mathematical Research

Universiti Putra Malaysia

43400 UPM Serdang, Selangor Darul Ehsan

Malaysia
Athirah Nawawi*

Department of Mathematics, Faculty of Science

Universiti Putra Malaysia

43400 UPM Serdang, Selangor Darul Ehsan

Malaysia

*Corresponding author; email: athirah@upm.edu.my

Received: 1 April 2020

Accepted: 4 August 2020 